Rabaska

Revue d'ethnologie de l'Amérique française

\title{
Michel Faubert
}

Parcours d'un artiste de scène

\section{Benoît Maheux}

Volume 3, 2005

URI : https://id.erudit.org/iderudit/201711ar

DOI : https://doi.org/10.7202/201711ar

Aller au sommaire du numéro

\section{Éditeur(s)}

Société québécoise d'ethnologie

ISSN

1703-7433 (imprimé)

1916-7350 (numérique)

Découvrir la revue

Citer ce document

Maheux, B. (2005). Michel Faubert : parcours d'un artiste de scène. Rabaska, 3, 71-81. https://doi.org/10.7202/201711ar d'utilisation que vous pouvez consulter en ligne.

https://apropos.erudit.org/fr/usagers/politique-dutilisation/ 


\section{Portrait}

Michel Faubert

Préparé par Benoît MaheuX

Université Laval

\section{Notice biographique}

Michel Faubert est né en 1954, à Rigaud, non loin de Montréal. Autodidacte, Faubert collectionne des chansons, des contes et des légendes en parcourant le Québec et l'Acadie depuis plus de vingt-cinq ans. Mais il ne fait pas qu'emmagasiner ses récoltes. Les histoires qu'il entend des vieux qui savent conter, il les remanie, les rhabille selon la mode contemporaine. Allié à des musiciens issus du milieu de la musique actuelle de Montréal, il redonne vie au folklore en se réappropriant les chansons ou les contes sous des sonorités urbaines, très folk et blues, et même industrielles à l'occasion.

On l'a affublé de tous les noms : défricheur de mémoires, folkloriste post-nucléaire, ethnologue sur le tas, ramancheur de patrimoine, transmet-

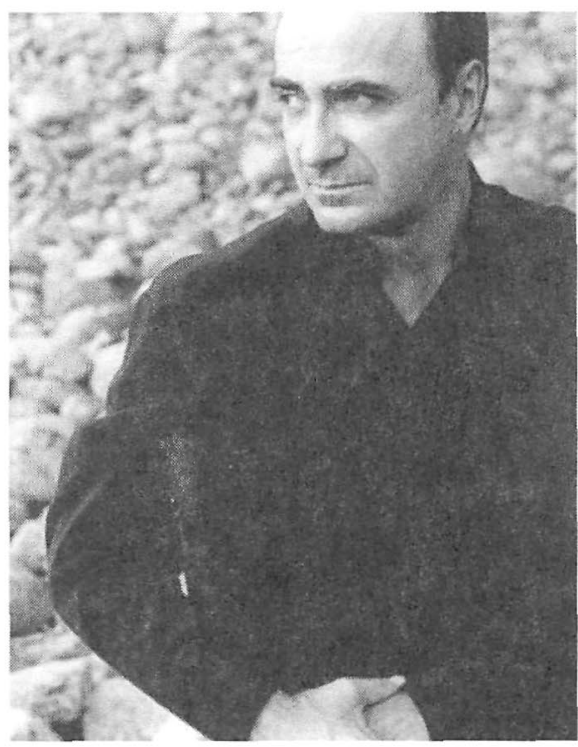
teur de mémoire. Mais Faubert se désigne sous les termes plus simples de conteur et chanteur. Il s'est créé un répertoire folklorique. À partir de ses collectes, il a enregistré trois disques de chansons : Maudite mémoire en 1992, Carême et Mardi gras en 1995 puis L'Écho des bois en 1997. En même temps et de la même manière, il a créé un disque de contes, Le Passeur. Parallèlement, avec le groupe les Charbonniers de l'enfer, ensemble vocal a cappela dont il était membre avec Michel Bordeleau, André Marchand, JeanClaude Mirandette et Normand Miron, il a participé à un album éponyme en 1993. Plus tard, en 2000, Faubert revenait à la chanson a capella avec l'album solo La Récompense. 
Changeant sa manière de travailler, il s'est mis à écrire ses propres contes par la suite et en publia le résultat dans L'âme qui sortait de la bouche du dormeur, en 2000. Chaque sortie d'album est suivie de tournées de spectacles, ce qu'il préfère, qui l'ont conduit du Québec, en passant par la France, jusqu'à Madagascar où il remporta la médaille d'or des Jeux de la francophonie.

Fin 2003, il a créé un nouveau spectacle Histoires sorcières, inspiré du répertoire de contes et légendes de l'ethnologue français Claude Seignolle, en duo avec Jérôme Minière. Aussi, les Charbonniers de l'enfer produisirent un deuxième album, $W \hat{o}$, toujours composé de chansons a capella et ils terminèrent leur tournée en février 2005. Polyvalent, créatif et prolifique, Michel Faubert continue à se mettre en bouche des histoires et des chansons pour vivre. 


\section{Parcours d'un artiste de scène $e^{1}$}

« C'est l'histoire d'un gars qui, un jour, est parti chez la dame Marie-Rose. Il pourrait s'appeler Ti-Jean, il pourrait s'appeler Michel. La dame ouvre la porte, le regarde... Puis, en le laissant entrer, elle s'informe de sa mère, elle s'informe toujours de sa mère. Lui, ce qu'il veut, c'est des chansons... et la dame en a, elle en a plein : des chansons, des complaintes, des histoires. Elle en avait plein, tout plein de petits cahiers griffonnés. Alors, le gars s'assoit et l'écoute... " Ici, paraphrasant à la manière d'un conte, Michel Faubert explique, dans Mers et montagnes, recueil publié en 2002, son premier contact avec le répertoire folklorique.

Michel Faubert vient de Rigaud, seul garçon d'une famille de filles dont il est le cadet. C'était un enfant solitaire « qui a beaucoup vécu de choses dans le secret des choses ${ }^{2}$ ». Et il s'est rendu compte dernièrement qu'un des thèmes récurrents de son répertoire de contes est le secret. « Les histoires fantastiques que je raconte maintenant reflètent la vie obscure des gens où il y a des sens cachés. Même pour moi. Et je n'ai pas fini de découvrir à quel point les contes parlent de nous-même ${ }^{3}$ ».

Vers la fin des années 1970, alors qu'il y avait une vague de folklore avec La Bottine Souriante et Garolou et « que les jeunes redécouvraient la musique traditionnelle 4 », il s'est mis à avoir un penchant vers le fantastique et le folklore. Il jouait déjà du violon et sa mère l'a mis en contact avec une de ses amies, Marie-Rose d'Amour. Faubert lisait Edgar Allan Poe, et la dame lui racontait des histoires de feux-follets et des complaintes d'autrefois;

1. Merci à Suzy Turcotte, mine de trésor incroyable, pour sa générosité et sa disponibilité. Elle est notre plus grande source pour l'élaboration de cet essai biobibliographique. Elle a interrogé Michel Faubert trois fois, depuis Maudite mémoire, et a accumulé un dossier de presse surprenant. Ces entrevues ont été diffusées à la radio communautaire, CKRL-MF, 89,1, à Québec. Les citations tirées des enregistrements de $M^{\text {me }}$ Turcotte sont identifiées $S$. Turcotte 1 pour l'entrevue réalisée en 1996, S. Turcotte 2 pour celle faite en 2000 lors du spectacle $L$ 'âme qui sortait pas la bouche du dormeur, et $S$. Turcotte 3 pour la plus récente, en octobre 2003, effectuée pour le spectacle Histoires sorcières. Chaque entrevue est d'une durée d'une demi-heure et est conservée dans la collection privée de Suzy Turcotte.

2. S. Turcotte $2,2000$.

3. S. Turcotte 3, 2003 .

4. S. Turcotte 2,2000 . 
elle « chantait des légendes 5 ». Elle en avait des cahiers pleins. La première chanson qu'elle lui chanta, La Fille du boulanger, touchera l'un des thèmes privilégiés de Faubert et qu'il considère nécessaire d'exploiter lorsqu'on fait dans le traditionnel : la mort. Cette certitude restera en lui tout le long de son parcours : «Elle est le premier mystère des petits enfants et la dernière compagne des vieillards laissés pour compte. Je la chante. Je la respecte. Et tout amant de la vie que je suis, j'ai peur de dire que je l'aime un peu... Pour le merveilleux qu'elle sème sur les terres qu'elle fauche ${ }^{6} \gg$.

Durant la semaine qu'il apprend la chanson, il visionne le film La Source de Bergman qui lui révèle que des parentés existent entre une chanson traditionnelle de son village et une légende scandinave du Moyen-Âge. Comme si le génie d'un peuple s'était étendu à plusieurs peuples... C'est l'été suivant, grâce à un projet étudiant, qu'il agrandit son répertoire de chansons folkloriques en allant fouiller dans tous les autres villages de son coin. Il est attiré par les complaintes aux textes forts, aux thématiques religieuses et merveilleuses.

Au début de 1980, il déménage à Montréal et développe des liens avec des musiciens de la scène underground, dont André Duchesne, guitariste de musique actuelle. De cette collaboration, naît un premier album, salué par la critique, Maudite mémoire, un album racontant la guerre et autres tragédies sur des airs de berceuses anciennes, sur une musique contemporaine bien différente de tout ce qui se produisait alors dans le «folklore » qui, selon Faubert, se limitait surtout aux chansons à boire et aux airs du temps des fêtes.

Faubert a orchestré et électrifié le son des complaintes. "La singularité de la démarche tenait à l'actualisation avant-gardiste d'un répertoire de complaintes... médiévales! [...] ces complaintes avaient toutes été conçues avant la découverte du Nouveau Monde ${ }^{7} »$, même si Faubert les a toutes recueillies au Québec et en Acadie. Ces complaintes ont suivi les pionniers, quelques-unes se sont adaptées au territoire américain, d'autres ont changé de nom. Le Prince Eusèbe est généralement intitulé Le Prince Eugène. Dans le livret de 1'album Maudite mémoire, Faubert explique la source de ses chansons et dans quelles circonstances il les a apprises, laissant ainsi voir ses efforts de collectes. Robert Bouthillier, ethnologue et musicien folkloriste, l'a accompagné longtemps dans ses démarches.

Puis Michel Faubert a travaillé deux ans avec un groupe de musiciens, dont Michel Langevin, de Voivod, donnant plus d'énergie musicale à son

5. S. Turcotte 1, 1996.

6. Michel Faubert, «L'Artiste et la mort ; esquisses d'autoportraits », dans Frontière, Québec, vol. 9, nº 1 , printemps-été 1996, p. 28.

7. Alain Brunet, La Presse, Montréal, 18 novembre 1995. 
deuxième album Carême et Mardi gras. " [Mon approche du métier] a changé dans le sens qu'au début c'était vraiment un travail de moine, je faisais les choses de mon côté, de la cueillette de chansons. Ça m'a pris beaucoup de temps à accumuler le matériel, et je n'étais pas prêt à le chanter, à le diffuser. Et le travail en groupe, avec Carême et Mardi gras a déclenché beaucoup plus de sûreté. [...] Depuis un an, un plaisir s'est ajouté à ça, il y a le métier qui rentre, mais aussi les voyages en France ${ }^{8}$ ").

Si Maudite mémoire est un disque introverti, le deuxième est beaucoup plus moderne, mais toujours basé sur les complaintes recueillies. À ce propos, Faubert dit : " Je marche sur une drôle de frontière. D'un côté, je me sens attiré vers le rock, mais plus je m'en approche, plus quelque chose en moi me tire vers le côté traditionnel. [...] Je ne veux pas, ne peux pas séparer les deux. [...] Quand je chante des trucs plus rock, j'entends cette voix, celle d'un chanteur de complaintes ${ }^{9}$ ».

Pour le spectacle de Carême et Mardi gras, six musiciens sont présents sur scène et Faubert appellera cela du « folklore vivant » parce que beaucoup plus contemporain.

Quand, en 1971, Philippe Gagnon et Dominique Tremblay, un violoniste classique et un violoneux, produisent Ça roule avec le stainless steel, un album folklorique avec des éléments psychédéliques, ils ont touché Faubert et leur style mélangé l'a grandement influencé. Sur ce mélange des genres musicaux, Faubert a remarqué qu'« il y a tout un courant de métal qui était inspiré des légendes. [...] Toute l'influence satanique du métal est la même qui est présente dans notre folklore ${ }^{10} 》$. Et ailleurs : « Aussi, dans le fond, les violoneux du temps, ceux qui prenaient un coup et couraient les filles, c'était des bums à leur façon. Ils avaient tous de la misère avec le curé ${ }^{11} »$.

Le métal satanique ou gothique contemporain a été influencé par les légendes d'autrefois. Faubert a été influencé par ce mélange des styles. Le groupe Le Rêve du diable fut, à son tour, influencé par Michel Faubert. Ce dernier dit être " tombé en bas de [s] a chaise quand [il a] entendu ça! $!^{12}$ » Et il ne commençait qu'à être connu.

Une thématique qui reviendra souvent chez Faubert est celle du religieux. Or, il fut étonné de la réticence de plusieurs de ses collaborateurs pour les chansons à caractère religieux. Pourtant, c'est cela qui amène le fantastique dans le folklore québécois avec le Yâb et le Bon Yeu. Mais Faubert ne s'en est pas formalisé pour autant. Son répertoire est resté touché par ce fantastique.

\footnotetext{
8. S. Turcotte 1, 1996.

9. Nicolas Tittley, Voir, Montréal, 16 au 22 novembre 1995.

10. Richard Labbé, Montréal Campus, Montréal, 6 au 19 septembre 1995, p. 20.

11. S. Turcotte $1,1996$.

12. S. Turcotte 2, 2000.
} 
En 1997, Faubert sort deux albums. Tout d'abord, L'Écho des bois, un album d'atmosphère qui évoque les voyages des gars de chantiers, la forêt magique ou bien celle tragique des songes. Un album complètement différent des deux autres avec les guitares omniprésentes, sans violon, très loin du " traditionnel ", du reel; un album folk, country, dans le style d'Abbitibbi, que la critique juge mature. Avec cet album, Faubert reçoit un Félix en 1998, pour «l'album folk de l'année » et il est aussi en nomination comme « interprète de l'année et spectacle de l'année ». Mais aussi, il a produit son premier album de contes, Le Passeur, qui transpose la veillée du conteur traditionnel par excellence, dont la forme classique apparait dans les contes de Ti-Jean. Seul le multi-instrumentiste Daniel Roy l'accompagnait sur scène et faisait le pont entre les contes. Avec ces deux albums, Faubert est invité dans plusieurs festivals et gagne la médaille d'or aux Jeux de la francophonie à Madagascar.

Au cours de son travail de collecte, Lucille Guilbert de l'Université Laval lui présente Ernest Fradette, un conteur qui ne se prétendait pas conteur, comme tous ceux des campagnes. Michel Faubert se lie d'amitié avec lui et sa femme, Yvette Doiron, qui avait en quelque sorte une fonction de mémoire. Elle connaissait les contes de son homme tout autant que lui. En fait, même Luc Lacourcière avait enregistré les contes de la famille Fradette et avait constaté que, lorsque le conteur avait un trou de mémoire, c'était sa femme qui continuait l'histoire. Faubert raconte : «Quand j'allais le voir, on jasait de choses et d'autres, et, à un moment donné, Ernest Fradette écrasait sa cigarette, se retournait d'un geste brusque et disait en me regardant droit dans les yeux : "C'est comme une fois... » et ne me lâchait plus des yeux jusqu'à la fin de l'histoire. Ça me clouait sur la chaise. C'est peut-être ce que j'ai retenu le plus ${ }^{13}$ ".

Parce qu'au fil de ses spectacles, Michel Faubert a pris de l'assurance. Rares sont les conteurs qui peuvent apprendre d'un « maître » comme il le fit. La cueillette de contes traditionnels, au Québec dans la famille Fradette, et en Acadie avec Robert Bouthillier, lui a aussi permis de rencontrer Honoré et Alvina Saint-Pierre, d'autres « informateurs », d'autres conteurs. Mais c'est à Saint-Raphaël-de-Bellechasse qu'il a appris l'art de conter, d'en faire une performance. « Ernest Fradette a trois sortes d'histoires : il conte des contes appris de son père, qu'il essaie de restituer. Il conte aussi des faits qu'on lui a relatés quand il était jeune. Et il conte à partir de ses propres souvenirs. Ce qui est fascinant chez lui, c'est que les souvenirs et les faits prennent l'allure, la forme et l'imagerie du conte. Le conte, je l'ai compris avec lui, c'est l'art de faire surgir des images ${ }^{14} »$. Les salles de ses spectacles étaient remplies,

13. Sylvain Cormier, Le Devoir, Montréal, jeudi 14 décembre 2000, p. B7. 
surtout de jeunes. Au début de ses performances, Faubert parlait sans cesse, car il avait peur des silences et croyait que « les salles combles étaient dues au débit de parole ${ }^{15} »$. Mais le métier aidant, il sut maîtriser les silences qui sont tout autant significatifs que les mots. "Les contes sont à la parole ce que la légende est au silence ${ }^{16} \%$.

À cette époque (1997), il ne savait trop s'il allait écrire ses propres légendes. Il chantait aussi avec Les Charbonniers de l'enfer, groupe traditionnel a capella et, ici encore, l'harmonie vocale était nouvelle, les chansons reprises n'avaient pas cette forme à prime abord. En 2000, dans un album solo, La Récompense, il reprend des complaintes de ses anciens albums, mais, cette fois, chantées dans leur forme traditionnelle.

Puis, en 2000, il produit un deuxième album de récits, L'âme qui sortait par la bouche du dormeur. Ce ne sont pas des contes, en fait, ce sont des légendes. «Les contes, c'est une histoire inventée. [...] C'est pour le plaisir et l'émerveillement. Tout le monde sait que c'est de la menterie. La légende, elle, est de l'ordre du communautaire [...] elle circule dans l'inconscient collectif, mais possède une résonnance profonde en chaque individu puisqu'elle se nourrit de nos douleurs et de nos peines... ${ }^{17}$ "

Pour la première fois, avec ce disque, Faubert signe les textes des légendes racontées. Avec Le Passeur, il racontait des contes récoltés un peu partout dans la province et disait qu'il ne savait trop si un jour il en écrirait. L'idée a fait son chemin. "Je suis en transition, je ne veux plus être un conteur folklorique. Je reste depuis vingt et un ans à Montréal et Montréal est entré dans le corps du folkloriste ${ }^{18}$ ». C'est ce qui le caractérise en fait : être un touche-à-tout, faire ce qui lui plaît. Il se sent loin d'être l'ethnologue sur le tas ou le gardien du patrimoine. Il vit en équilibre entre la complainte et la légende. Avec ce spectacle, il a cimenté la musique et l'histoire, il y a même des pièces musicales où il raconte mais, dit-il : «Je ne sais pas si j'écrirai un jour des paroles sur des complaintes, mais c'est normal que je me sois d'abord essayé avec le conte. C'est la parole avec laquelle je me sens le plus libre ${ }^{19}$ ". Le conte La Vendeuse de rêves s'inspire directement d'un de ses souvenirs d'enfance. C'en était un qui le hantait; il a menti à son père prenant sa Pontiac Parisienne pour aller voir les danseuses. C'était sa chasse-galerie à lui, avec son char-canot. "Les souvenirs personnels qu'on a finissent par prendre l'allure des contes qu'on raconte. Et on confond les éléments de la réalité
14. Loc. cit.
15. S. Turcotte $2,2000$.
16. Loc. cit.
17. Éric Moreault, Le Soleil, Québec, 15 janvier 2000, p. 12.
18. S. Turcotte 2, 2000.
19. Sylvain Cormier, Le Devoir, Montréal, jeudi 14 décembre 2000, p. B7. 
avec des éléments de fiction commune, une mémoire collective $\mathrm{e}^{20} »$. On voit ici que la démarche du conteur, inaugurée par les contes traditionnels, a pris une tangente bien différente : «Une légende, c'est plus qu'une histoire, c'est une vision, ça se passe à un moment où on est fébrile, en état de danger ${ }^{21}$ ».

Depuis le début de sa carrière, ce qu'il aime depuis toujours, ce sont les spectacles. Le spectacle pour lui est une forme de thérapie collective et il décrit le conteur comme un "médecin de l'âme ${ }^{22}$ ». Il fait le métier qu'il aime et sa vie prend son sens, sur scène, dans le rapport avec les autres. La performance est donc très importante pour capter le public, le garder avec soi et l'amener ailleurs, là où il affronte ses angoisses dans la légende. " Lorsque j'écoute un conteur, il m'arrive de décrocher complètement, parce que je me laisse bercer par la musique de la voix, parce que je regarde ses mains, son corps, etc. Pour le conteur, il y a ce plaisir de la gestuelle, de la musique des mots $^{23}$ ». Il faut avoir une prestance forte pour pouvoir regarder le public dans les yeux.

Mais Michel Faubert n'est pas le seul conteur au Québec. Certes il est bien connu, quelques journalistes le consacrent vedette de la scène underground, conteur national, mais il y en a plusieurs autres qui émergent, et, eux non plus, ne restent pas campés dans la forme du conte traditionnel. Les bars Le Sergent recruteur à Montréal et le Fou bar à Québec ont eu une grande influence sur cette forme de spectacle. Ils donnaient une scène aux conteurs et ceux-ci avaient une visibilité possible. Le regain de popularité des contes depuis plusieurs années est aussi dû au Festival interculturel du conte de Montréal, et aux Contes urbains de Montréal et de Québec. «C'est bien l'attrait que peut susciter cette musique-là [la traditionnelle], et le conte aussi; il y a vraiment quelque chose qui se passe au Québec et en Occident en général, qui n'existait pas il y a dix ou quinze ans ${ }^{24} »$. Pour la musique, il y a Les Batinses, groupe de Québec qui a commencé, comme Faubert, à chercher dans les Archives de folklore de 1'Université Laval. Ils ont chanté des complaintes sur fond de musique traditionnelle avec violon, podorythmie et bombardes ${ }^{25}$, mais, maintenant, après trois albums, ils versent dans le funk, ou le trash-ditionnel, toujours avec des chansons prises aux Archives de

20. S. Turcotte $2,2000$.

21. Loc. cit.

22. Loc. cit.

23. Laurent Saulnier, Voir, Montréal, 29 février au 6 mars 1996.

24. S. Turcotte $1,1996$.

25. Ou guimbarde. « Si vous trouvez que l'appellation « guimbarde» rend bien peu justice à la grandeur d'un instrument qui fut autrefois aussi sauvage qu'envoûtant, et si vous pensez comme nos grands-parents que le terme « bombarde » serait plus approprié, vous pouvez témoigner de votre appui en écrivant au Front de Libération de la Bombarde, 266 Arago, Québec, G1H 2H8 ». Post-scriptum dans le livret de l'album Le Passeur. 
folklore, et quelques compositions de leur cru. « Ça me fait plaisir de voir des nouveaux conteurs comme Simon Gauthier et Fred Pellerin qui développent leur propre démarche ${ }^{26} \gg$. Et il y a aussi Marc Laberge, André Lemelin et Jojo Turenne. Suzy Turcotte, en entrevue ${ }^{27}$, fait voir à Faubert qu'il peut être devenu le passeur pour une nouvelle génération, reprenant le thème du premier disque de contes. Faubert prend conscience de sa responsabilité mais ne s'inquiète pas, ne s'en fait pas : «Ça me stimule, je dois me situer, et aussi ça m'enlève un poids. Ça me permet de faire mes affaires $^{28}$ ». Plus de conteurs, c'est aussi plus de voix qui éclatent dans ce monde : "Les conteurs ont une liberté que peu d'écrivains se permettent d'avoir. On est dans l'univers du politically correct $^{29}$ ", et le conteur, lui, peut tout transgresser.

Maintenant, Faubert a pris encore un autre tournant, la réalité a dépassé la fiction ou, plutôt, le passé l'a d'une certaine manière rattrapé. S'il lisait Edgar Poe quand il était tout jeune, les récits véritablement fantastiques sont revenus le hanter avec son tout dernier spectacle, Histoires sorcières. Il le fait en duo avec Jérôme Minière, inspiré du répertoire de l'ethnologue français Claude Seignolle. Ce dernier, qui a connu Jean Bergier, celui qui a écrit le Matin des Magiciens avec Louis Pauwel, a à son tour écrit Les Évangiles du Diable et une Anthologie des croyances sur le diable. Quatre-vingt-cinq ans, " homme étrange et drôle ${ }^{30}$ », Seignolle a appelé Faubert et ils se sont rencontrés à Paris, dans sa bibliothèque. Seignolle lui a proposé de se réapproprier les histoires qu'il a lui-même recueillies, en France, dans le Périgord entre autres. « Les histoires de Seignolle se passent à la campagne, majoritairement, elles sont faciles à adapter en milieu québécois. Les histoires parisiennes sont plus difficiles ${ }^{31}$ ». Ainsi, Faubert a développé une autre démarche pour ce spectacle. Privilégiant les rencontres, il a invité Jérôme Minière à se joindre à lui, pour qu'il fasse sa musique électronique minimale sur les contes. Mais Faubert lui a demandé de conter avec lui, Minière ayant déjà un regard insolite dans ses chansons du quotidien. Histoires sorcières est donc un spectacle différent encore de tout ce qu'il a fait jusqu'à présent. Faubert et Minière ont écrit des légendes ensemble, inspirées de celles de Claude Seignolle, mais «l'important, vraiment, c'est le fantastique ${ }^{32}$ ».

26. S. Turcotte 3, 2003.

27. S. Turcotte 2, 2000 et S. Turcotte 3, 2003.

28. S. Turcotte 3, 2003.

29. Loc. cit.

30. Loc. cit.

31. Loc. cit.

32. Loc. cit. 
Donc, à l'instar de folkloristes comme Marius Barbeau, Faubert n'a pas voulu créer un folklore québécois. Il n'y a pas de nationalisme là-dedans. Il n'a pas voulu porter une ceinture fléchée et taper du pied en disant : «Et swignez la compagnie! » Même s'il chantait des complaintes anciennes et jouait quelques reels aussi, il actualisait tout ce qu'il a recueilli. Las de se faire surnommer ou étiqueter comme le sauveur du patrimoine, il a bifurqué dans son approche du métier. Il a voulu diffuser ce qu'il avait déniché dans le folklore au moyen de sa cueillette. Et il a découvert ce qu'il aime : c'est la scène, c'est conter, c'est chanter. Alors il fait ce qu'il veut. S'il situe ses histoires au Québec, c'est pour qu'elles soient plus près de son auditoire. Parions que, $\mathrm{s}^{\prime}$ il conte en France, il peut très bien replacer sa légende dans le champ à côté de la salle de spectacle...

C'est ce qui est intéressant avec les conteurs. Comme le disait Faubert, ils s'offrent la liberté de faire ce qu'ils veulent. L'ethnologie étant une science dynamique, le rapport à l'autre étant très important, elle l'est aussi pour la performance du conte. Si les gens rient à un endroit où le conteur ne veut pas qu'ils rient, il se rajuste la fois suivante. C'est mouvant et chaque interprétation est différente. Or, Michel Faubert a entendu dire qu'un jeune contait L'Argent de mon cochon exactement comme sa version enregistrée du disque $L e$ Passeur. Lui, " qui connaissai[t] par cœur tous les monologues d'Yvon Deschamps ${ }^{33} »$, il s'est rendu compte de la portée de ses paroles.

Puisqu'on ne peut pas le nier, Faubert tout d'abord folkloriste autodidacte et « ethnologue sur le tas » est maintenant devenu, avant tout, un artiste de la scène. Un homme qu'on veut aller voir en spectacle et qui va raconter des peurs et des menteries. Il a du respect pour ceux qui cherchent encore en lui l'ange-gardien du patrimoine, mais il s'efforce de protéger sa liberté d'aller où il veut. Avec Les Charbonniers de l'enfer, il canalise encore des chansons traditionnelles mais, pour ce qui est du conte, de la légende, il est, comme sa voix, libre. « Je ne veux plus m'enraciner uniquement dans des racines du Québec. [Avec Histoires sorcières], j'ai l'impression que je viens de m'ouvrir à plus de possibilités que je ne me permettais pas avant, plus universel. Les histoires peuvent être n'importe où. Avant, c'était plus important de les situer. C'était important pour moi de savoir qu'à Rigaud, il y avait une chanson ancienne rattachée à une légende scandinave. Mais c'est pas nécessaire de fouiller dans l'histoire pour trouver; les univers peuvent se toucher. Maintenant je peux prendre une légende scandinave, l'adapter et la raconter comme si elle se passait ici ${ }^{34} »$.

33. S. Turcotte 2, 2000.

34. S. Turcotte 3, 2003. 
Michel Faubert a suivi un parcours tortueux. Il est arrivé à un carrefour, au milieu du village. Il a été voir tous les chemins possibles et il a emprunté ceux qui menaient aux bois, qu'il a traversés. Il est revenu au carrefour pour repartir ensuite, tentant de toucher à tout ce dont il avait envie. Car il veut rester toujours libre de ses choix, authentique dans sa carrière, et indépendant dans ses histoires. Et sans le vouloir, il a fini par devenir " passeur » d'un goût de conter, au Québec, à une nouvelle génération.

\section{Publications de Michel Faubert}

\section{Disques}

\section{a) Discographie personnelle}

Maudite Mémoire, Montréal, Ambiance magnétique, 1992 (chansons). Carême et Mardi gras, Montréal, Milles-Pattes, 1995 (chansons). L'Écho des Bois, Montréal, Milles-Pattes, 1997 (chansons). Le Passeur, Montréal, Mille-Pattes, 1997 (contes). La Récompense, Montréal, La Tribu, 2000 (chants a capella solo). L'âme qui sortait par la bouche du dormeur, Montréal, La Tribu, 2000 (contes).

\section{b) Discographie en collaboration}

Musique Multi-Montréal Montréal, Disque MMM, 1994 (chanson avec Karen Young).

Les Charbonniers de l'Enfer, Montréal, Milles-Pattes, 1996 (groupe de chants a capella avec André Marchand, Normand Miron, Jean-Claude Mirandette et Michel Bordeleau).

Daniel Roy / A u tour du flageolet, Montréal, Mille-Pattes, 1996 (chanson). Henri Band / Henri III, Montréal, Disque MPV, 1998 (chanson rock).

WD 40 / Aux frontières de l'asphalte, Montréal, Disque MPV, 1999, (chanson rock).

Papa Boa / Tête à queue, Montréal, Ambiance magnétique, 1999 (musique actuelle).

Un trésor dans mon jardin, Montréal, La Montagne secrète et Édition Le vent qui vire, 2002 (chansons, contes et comptines de Gilles Vigneault).

Les Charbonniers de l'Enfer, Wô, Montréal, La Tribu, 2002 (chanson a capella).

\section{LIVRE}

Mers et montagnes, Trois-Pistoles, Éditions Trois-Pistoles, 2002, 99 p. (anthologie de chansons collectées). 